\title{
NUEVOS DATOS SOBRE EL GÉNERO Sucocystis (CINCTA, ECHINODERMATA) EN EL CÁMBRICO MEDIO DE ESPAÑA: IMPLICACIONES BIOSTRATIGRÁFICAS Y FILOGENÉTICAS
}

\author{
Samuel ZAMORA e Imran A. RAHMAN \\ ${ }^{1}$ Área y Museo de Paleontología. Departamento de Ciencias de la Tierra. Uni- \\ versidad de Zaragoza. E-50009 Zaragoza. España. samuel@unizar.es \\ ${ }^{2}$ Department of Earth Science and Engineering, Imperial College London, \\ London SW7 2AZ, United Kingdom. i.rahman@imperial.ac.uk
}

Zamora, S. \& Rahman, I. A. 2008. Nuevos datos sobre el género Sucocystis (Cincta, Echinodermata) en el Cámbrico medio de España: Implicaciones biostratigráficas y filogenéticas. [New data on the genus Sucocystis (Cincta, Echinodermata) from the middle Cambrian of Spain: Biostratigraphic and phylogenetic implications.] Revista Española de Paleontología, 23 (2), 301-313. ISSN 0213-6937.

\begin{abstract}
New material of the carpoid class Cincta is described from the middle Cambrian of the Iberian Chains (Spain). Material consists of the dorsal surface of a well preserved individual belonging to the genus Sucocystis; this specimen comprises a complete theca and a partial stele. The marginal ring (cinctus) is composed of twelve marginal plates (M6r-M51). Two well-defined grooves run from the mouth around the outer face of the marginal ring: the right groove is short, terminating in M2r; the left one is longer, ending in the medial part of M21. The lintel is composed of four suropercular plates, which are larger than other supracentral ossicles. The shape of the theca, length of the left marginal groove and structure of the lintel allow Sucocystis sp. A to be differentiated from other members of the Sucocystidae. The specimen was found in Mesones de Isuela (Zaragoza, Spain), in levels from the upper part of the Murero Formation (lower Languedocian), which correspond to the Solenopleuropsis thorali zone. It is one of the oldest representatives of the genus and expands the stratigraphic range of Sucocystis in the middle Cambrian of Spain. Sucocystis sp. A is unique among the Sucocystidae in the length of the left marginal groove, which reaches plate M2l. Comparisons with other species of Sucocystis (sensu Friedrich, 1993) demonstrate that there was considerable variation in the length and number of food grooves within the genus, suggesting that Sucocystis may be a paraphyletic group.
\end{abstract}

Key words: Cincta, Echinodermata, Sucocystis, middle Cambrian, Spain, biostratigraphy, phylogeny.

\section{RESUMEN}

Se describe nuevo material de carpoideos de la clase Cincta del Cámbrico medio de las Cadenas Ibéricas (España). El material se compone de un ejemplar identificado como perteneciente al género Sucocystis. La longitud de los surcos epitecales y las características del dintel hacen posible diferenciar a Sucocystis sp. A del resto de los Sucocystidae conocidos. El ejemplar procede de la localidad de Mesones de Isuela (Zaragoza) y fue recogido en el techo de la Formación Murero en niveles de la zona de Solenopleuropsis thorali, de edad Languedociense inferior. Esto permite ampliar considerablemente el rango biostratigráfico de este género en nuestro país. La comparación con el material previamente descrito en otras regiones gondwanicas, indica que se trata de uno de los representantes más antiguos del género Sucocystis. Se analiza la importancia de Sucocystis sp. A en la evolución del grupo, ya que se trata del único representante de la Familia Sucocystidae cuyo surco epitecal izquierdo alcanza la placa M21. Tras comparar la longitud de los surcos epitecales en las distintas especies del género (sensu Friedrich, 1993) se observa una variación importante en número y longitud de los mismos. Esta variación hace proponer a Sucocystis como un grupo parafilético.

Palabras clave: Cincta, Echinodermata, Sucocystis, Cámbrico medio, España, biostratigrafía, filogenia. 


\section{INTRODUCCIÓN}

Los cincta son un grupo de equinodermos primitivos que aparecen únicamente en rocas del Cámbrico medio. Su esqueleto se compone de multitud de placas calcíticas ensambladas entre si y con una estructura tridimensional denominada estereoma que, entre los filos actuales, es exclusiva de los equinodermos (Bottjer et al., 2006). El cuerpo de los cincta se compone de dos partes principales, teca y estela. La teca está delimitada por una serie de grandes placas que forman un anillo rígido llamado cinctus o marginalia. Esta estructura se encuentra cubierta, dorsal y ventralmente, por dos tegumentos de pequeñas placas ensambladas entre si que encierran la cavidad corporal. La estela es un apéndice caudal en forma de daga. Los cincta son organismos asimétricos y presentan una boca en la parte anterior derecha, a partir de la cual surge un sistema ambulacral, también asimétrico, compuesto por uno o dos surcos epitecales. En la parte anterior aparece una gran placa denominada opérculo, que es la principal característica diagnóstica, y cuya función ha suscitado importantes controversias (Jaekel, 1918; Ubaghs, 1968; Jefferies, 1990; Friedrich, 1993; Zamora et al., 2007a; Zamora \& Smith, 2008).

La extraña anatomía que presentan los cincta es la responsable de que su posición filogenética haya sido ampliamente discutida. Considerados primeramente como un grupo de equinodermos pedunculados (Barrande, 1887), actualmente se barajan tres hipótesis que los sitúan, bien como un grupo basal de hemicordados (Domínguez \& Jefferies, 2005), como un grupo de equinodermos blastozoos que secundariamente han perdido la simetría radial (Parsley, 1999; David et al., 2000) o como un grupo basal de equinodermos (Bather, 1930; Ubaghs, 1971, 1975; Jefferies et al., 1996; Smith, 2005; Zamora \& Smith, 2008; Rahman \& Zamora, en prensa).

El género Sucocystis es extremadamente raro en nuestro país y se encuentra representado por unos pocos ejemplares de una sola especie, Sucocystis melendezi (Schroeder, 1973), proveniente del Cámbrico medio (Languedociense superior) de la región de Ateca (Cadenas Ibéricas, Zaragoza).

El objetivo de este trabajo es describir un nuevo ejemplar perteneciente al género Sucocystis proveniente de una nueva localidad de las Cadenas Ibéricas. El fósil ha sido encontrado en el techo de la formación Murero en materiales de edad Languedociense inferior (Cámbrico medio). Este nuevo hallazgo permite ampliar considerablemente el rango estratigráfico de este género en España. Un análisis comparativo de esta nueva forma permite diferenciarla del resto de los Sucocystis conocidos, aunque ante la escasez de material disponible se prefiere dejar de momento el taxón en nomenclatura abierta. Además, se analiza la reducción, en número y longitud, de los surcos epitecales en las especies del género Sucocystis, y su significado en la historia evolutiva del grupo.

\section{SITUACIÓN GEOGRÁFICA Y GEOLÓGICA}

El material procede de la localidad de Mesones de Isuela situada a 75 kilómetros al OSO de Zaragoza (Fig. 1a). Los materiales cámbricos que aparecen en esta región se sitúan geológicamente en la parte norte de las Cadenas Ibéricas y más concretamente en la Unidad de Mesones (Gozalo \& Liñán, 1988). Esta Unidad, junto a la de Herrera y Badules, conforman la subdivisión del Paleozoico de las Cadenas Ibéricas (Lotze, 1929; Carls, 1983; Liñán, 1983; Gozalo \& Liñán, 1988).

Aunque los materiales cámbricos de la Unidad de Mesones son conocidos desde finales del siglo XIX y principios del XX (Donayre, 1873; Palacios, 1893; Lotze, 1929), fue Lotze (1961), quien presentó el primer esquema estratigráfico para la región. En él, se incluían unidades estratigráficas que abarcaban desde las Capas de Embid (Cámbrico inferior) hasta las Margas de Murero (Cámbrico medio). Lotze (1961) también incluye en su trabajo una lista de los fósiles recogidos en la Formación Huérmeda (Sdzuy, 1961). Aliaga (1968) en su tesis doctoral incluye la Unidad de Mesones y amplía su conocimiento estratigráfico y paleontológico. Schmidt-Thomé (1973) y Olmo et al. (1981) también trabajaron algunos aspectos geológicos de esta unidad. Valenzuela et al. (1990) realizaron un trabajo centrado en la Unidad de Mesones entre las localidades de Brea de Aragón y Mesones de Isuela. Este trabajo incluye una cartografía detallada y dos series estratigráficas de detalle, una en el Cámbrico inferior y otra en el Cámbrico inferior-medio. Los trabajos posteriores sobre la región se han centrado básicamente en la Formación Valdemiedes, en los niveles entorno al límite Cámbrico inferior-medio (Gozalo et al., 1993; Clausen, 2004; Dies Álvarez et al., 2004).

El ejemplar estudiado en este trabajo procede del techo de la Formación Murero (Fig. 1b) (Cámbrico medio) y su posición estratigráfica coincide con lo que Valenzuela et al. (1990) denominan tramo III de la Formación Murero. Se trata de un paquete constituido predominantemente por lutitas rojas aunque también aparecen algunos niveles intercalados de lutitas verdosas y algún nivel de areniscas. Valenzuela et al. (1990) identifican la presencia del trilobites Eccaparadoxides sp. Junto al carpoideo estudiado en este trabajo se recogieron además trilobites clasificados como Solenopleuropsis thorali Sdzuy, 1958, Solenopleuropsis marginata Sdzuy, 1958, Bailiella sp., Conocoryphe sdzuyi Courtessole, 1967, y Eccaparadoxides brachyrhachis (Linnarsson, 1883), que indican la Zona de Solenopleuropsis thorali, de la base del Languedociense inferior.

Otros equinodermos descritos en los afloramientos de Mesones de Isuela incluyen placas aisladas de eocrinoideos clasificados como Rhopalocystis? mesonesensis, Rhopalocystis? cf. mesonesensis y gen. et sp. indet. (Clausen, 2004) procedentes de la parte baja de la Formación Valdemiedes (Bilbiliense) y un edrioasteroideo clasificado 
como Cambraster cf. tastudorum (Zamora et al., 2007b) encontrado en la Formación Murero, en niveles de edad Caesaraugustiense superior.

\section{ANTECEDENTES SOBRE EL GÉNERO Sucocystis}

El género Sucocystis fue definido originariamente en Francia por Cabibel et al.(1959), quienes crearon la especie tipo Sucocystis theronensis a partir de tres ejemplares de la colección Miquel, en el Museo de Historia Natural de París. Trabajos posteriores consideraron a este género como sinónimo de Gyrocystis Jaekel, 1918 (ver Ubaghs, 1968). Sucocystis melendezi (Schroeder, 1973) fue la primera especie de este género definida en España. Aunque, originariamente fue asignada con dudas al género Gyrocystis por Schroeder, posteriormente se incluyó en el género Sucocystis por Friedrich (1993). Los ejemplares provenían de materiales del Languedociense, Cámbrico medio de las Cadenas Ibéricas y habían sido recogido años antes por Schmitz (1971) y Josopait (1972) durante sus respectivos trabajos de tesis doctoral.

Casi de manera coetánea al trabajo de Schroeder (1973), Termier \& Termier (1973) crearon en Francia el género y la especie Trochocinctus pardailhanicus (sinónimo subjetivo más reciente de Sucocystis melendezi). De acuerdo con Friedrich (1993), la prioridad de la definición de la especie corresponde al trabajo de Schroeder (1973) que fue publicado en el mes de Febrero, mientras que el trabajo de Termier \& Termier (1973) se publicó en diciembre.

Friedrich (1993) enmendó el género y estableció los criterios diagnósticos que permiten identificarlo. También definió varias nuevas especies: S. bretoni Friedrich, 1993 y S. acrofera Friedrich, 1993 de Francia y S. quadricornuta Friedrich, 1993 y S. undata Friedrich, 1993 de Marruecos. Además, asignó Cyclocinctus maroccanus a Sucocystis? maroccana (Termier \& Termier, 1973). En este mismo trabajo Friedrich creó la familia Sucocystidae. Una curiosidad de su trabajo, es que se realizó la reconstrucción de Sucocystis melendezi a partir de material proveniente del Norte de España encontrado por el Profesor Sdzuy. Este material nunca ha sido ilustrado, aunque Sdzuy (1993), en la introducción de su trabajo, indica que existe un manuscrito en preparación sobre esta especie que jamás fue publicado. Tras revisar las colecciones del Instituto de Paleontología de Würzburg (Alemania) se ha concluido que el material permanece allí pero carece de localidad exacta. Recien-

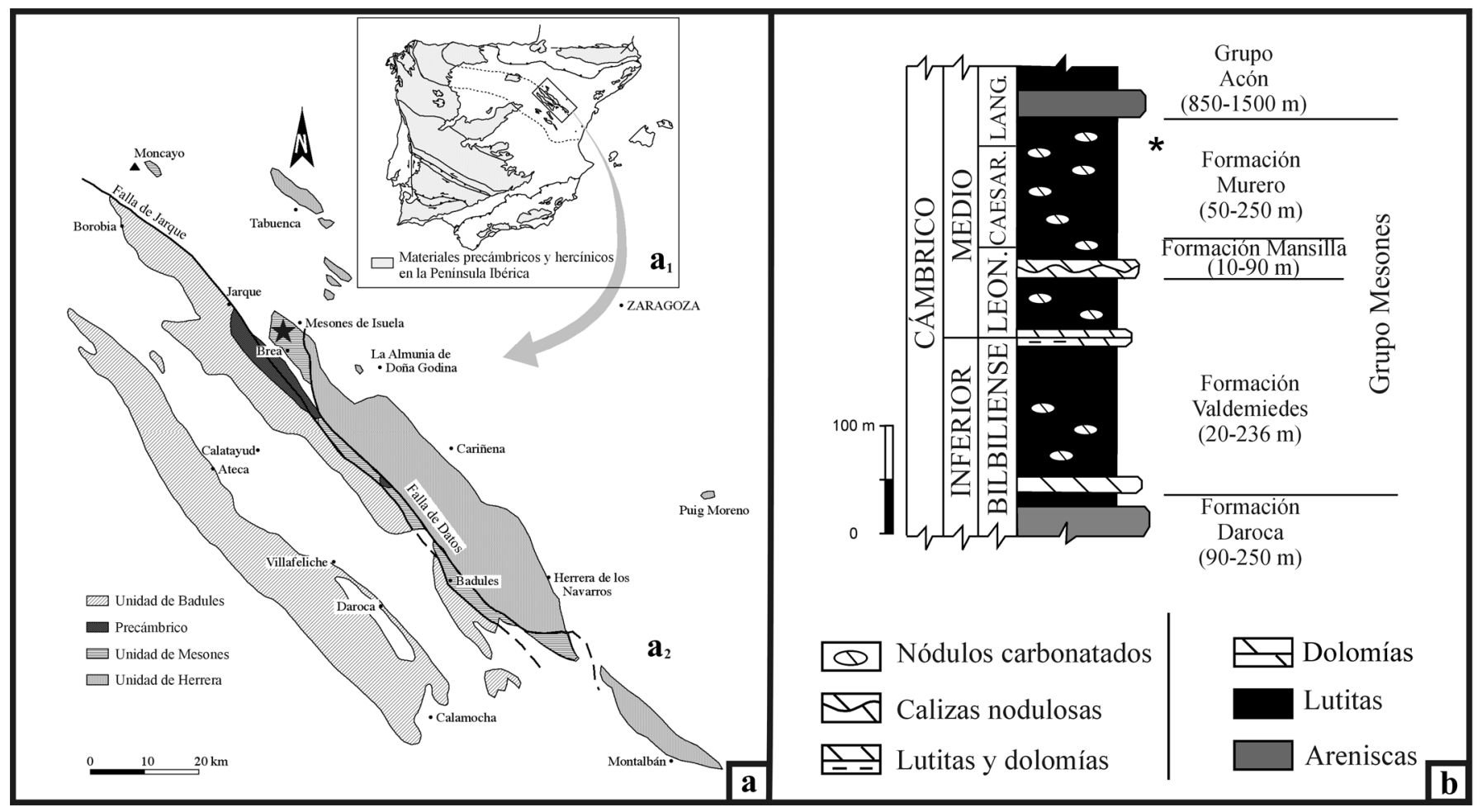

Figura 1. a, Situación geográfica y geológica de las Cadenas Ibéricas (según Gozalo \& Liñán, 1988); el material fue recogido, en las cercanías de Mesones de Isuela (provincia de Zaragoza). b, Columna estratigráfica del Grupo Mesones donde aparece la posición estratigráfica del material estudiado en el techo de la Formación Murero (según Liñán et al., 1996).

a, Geological and geographical setting of the Iberian Chains (after Gozalo \& Liñan, 1988); locality where material was collected, near Mesones de Isuela (Zaragoza province). b. Stratigraphic section of the Mesones Group with the horizon where Sucocystis sp. A. was recovered in the upper part of the Murero Formation (after Liñan et al., 1996). 
temente se ha podido constatar que este material procede del área de Ateca (Liñán, com. pers.)

Sdzuy (1993) discute la validez de la familia Sucocystidae Friedrich, 1993, y considera que los caracteres diagnósticos no son suficientes para diferenciarla de la familia Trochocystitidae, considerando a Sucocystidae como un sinónimo subjetivo más reciente de Trochocystitidae.

El último trabajo que trata el género Sucocystis se lo debemos también a Friedrich (1995). En él se cita por primera vez la presencia de este género en el Cámbrico medio de Cerdeña (Italia) y se discute sobre la validez de la familia Sucocystidae.

\section{PALEONTOLOGÍA SISTEMÁTICA}

El material está depositado en el Museo Paleontológico de la Universidad de Zaragoza con las sigla MPZ 2007/2479.
Material procedente de otras instituciones aparece siglado como:

NHM: Natural History Museum, London. UK.

PIW: Institut für Paläontologie der Julius Maximilians, Universität Würzburg. Alemania.

GPM: Geologisch-Paläontologisches Institut und Museum der Westfälischen Wilhelms, Universität Münster. Alemania.

IGUR: Institut de Géologie, Université de Rennes. Francia.

Metodología y terminología empleada: Todas las fotografías y dibujos a cámara clara han sido realizados a partir de moldes de látex blanqueados con cloruro de amonio.

Los términos empleados en este trabajo para la descripción se han tomado de Ubaghs (1968), Friedrich (1993) y Sdzuy (1993). Una traducción al español de una parte de ellos puede verse en Gil Cid \& Domínguez (1995) y Zamora et al. (2007a).

La nomenclatura de las placas marginales sigue la propuesta de Friedrich (1993) en la que la placa anterior que coincide con el plano axial se denomina M0. A partir de ella se numeran el

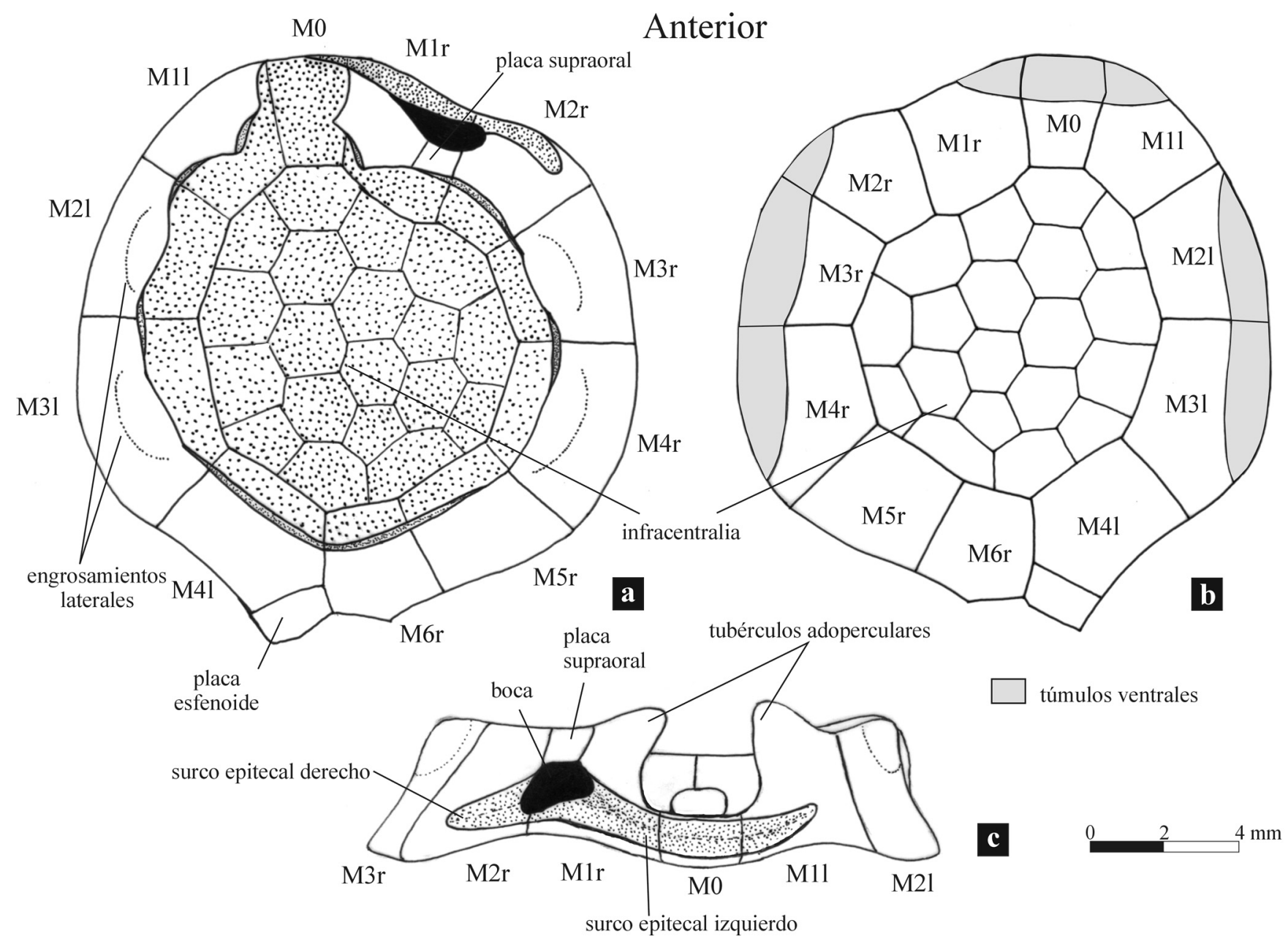

Figura 2. Reconstrucción de Sucocystis undata Friedrich, 1993 donde aparecen las principales partes anatómicas que pueden reconocerse en el género Sucocystis (modificada de Friedrich, 1993). a, dorsal; b, ventral; c, frontal. Abreviaturas: M, placa marginal; 1, izquierda; r, derecha.

Reconstruction of Sucocystis undata Friedrich, 1993 to show the anatomical terminology and orientation of the genus Sucocystis (modified from Friedrich, 1993). a, dorsal view; $\boldsymbol{b}$, ventral view; $\boldsymbol{c}$, frontal view. Abbreviations: M, marginal plate; l, left; r, right. 
resto según se encuentren a izquierda $(\mathrm{M} 11, \ldots, \mathrm{Mnl})$ o derecha (M1r, ..., Mnr) de ésta. Los términos anterior, posterior, lateral, izquierda, derecha, dorsal, ventral son utilizados de manera convencional según la posición de las principales aberturas (anterior) o de la posición de vida (cara inferior, ventral).

Clase CINCTA Jaekel, 1918

\section{Familia Sucocystidae Friedrich, 1993}

Discusión: Ubaghs (1968) en el tratado sobre paleontología de invertebrados considera la existencia de dos familias de cincta, Gyrocystidae Jaekel, 1918 y Trochocystitidae Jaekel, 1900. Friedrich (1993) creó la familia Sucocystidae donde incluye dos géneros Sucocystis y Elliptocinctus Termier \& Termier, 1973. Propone con reservas que Gyrocystidae y Sucocystidae puedan ser grupos hermanos. Sin embargo Sdzuy (1993) considera que Sucocystis es un descendiente de Trochocystites Barrande, 1859 y por tanto la familia Sucocystidae es sinónima de Trochocystitidae. La principal razón que Sdzuy (1993) da para esto es que en el género Sucocystis presenta una teca ligeramente asimétrica, y que esta característica se observa también en Trochocystites.
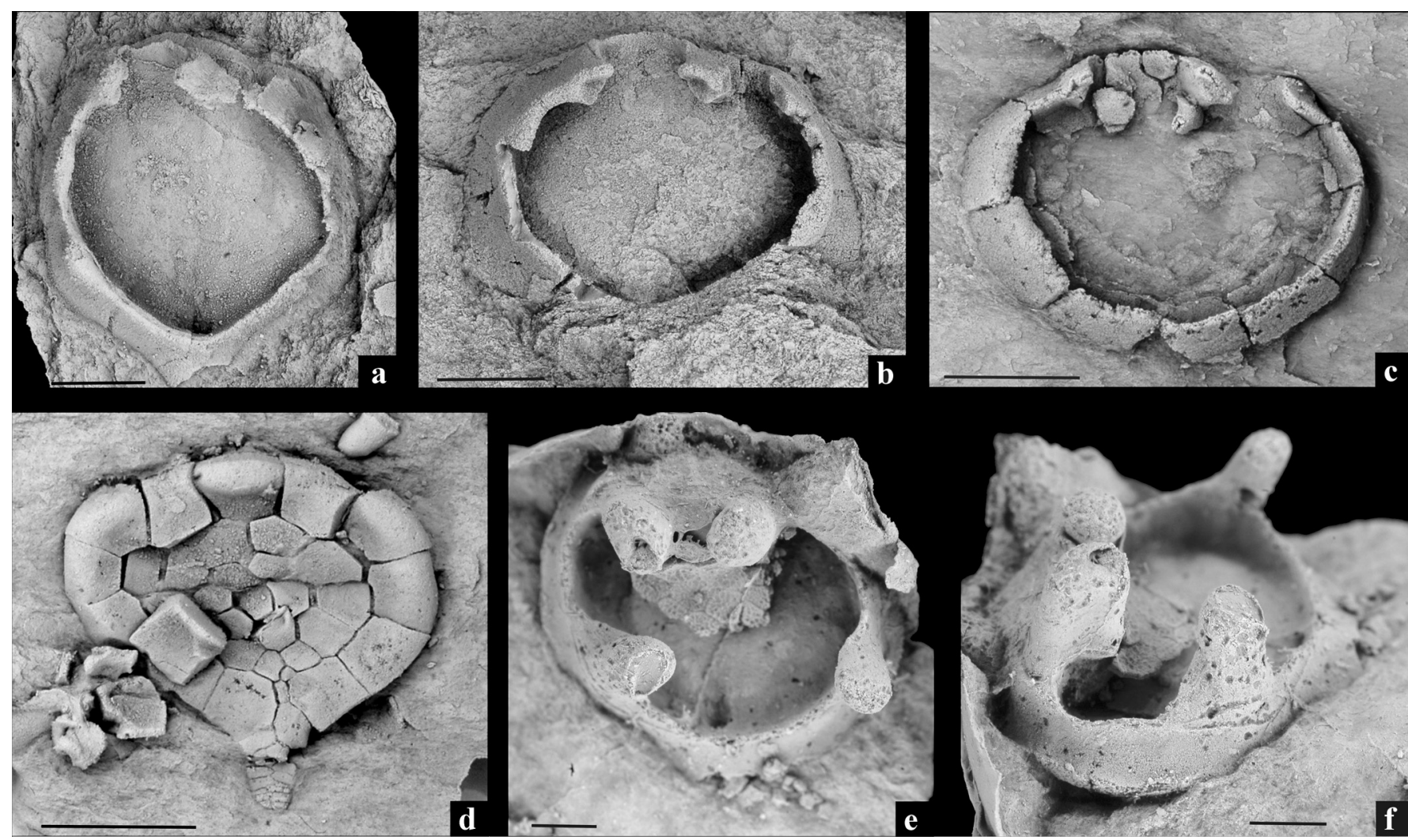

Figura 3. Fotografías de varias especies de Sucocystis donde se reconocen las principales características anatómicas del género. Todos los ejemplares han sido fotografiados a partir de moldes de látex teñidos con cloruro amónico. a: Cara dorsal de Sucocystis undata Friedrich, 1993, Holotipo IGUR 1033a, Cámbrico medio (Marruecos); observar los engrosamientos laterales en las placas marginales típicos de esta especie. Barra: $5 \mathrm{~mm}$. b: Cara dorsal de Sucocystis melendezi (Schroeder, 1973), Holotipo GPM L51/4/20, Cámbrico medio (Ateca, Zaragoza, España). Barra: 5 mm. c: Cara dorsal de Sucocystis acrofera Friedrich, 1993, PIW 92V183, Cámbrico medio (Montagne Noire, Francia). Barra: 5 mm. d: Cara ventral de Sucocystis theronensis Cabibel, Termier \& Termier, 1959, PIW 92V16II, Cámbrico medio (Montagne Noire, Francia). Barra: 5 mm. e, f: Cara dorsal y vista lateral de Sucocystis quadricornuta Friedrich, 1993, Holotipo PIW 92V51, Cámbrico medio (Maruecos), observar las grandes protuberancias sobre el cinctus diagnósticos de la especie. Barra: $2 \mathrm{~mm}$.

Different species of Sucocystis showing the characteristic features of the genus. All photographs are of latex casts taken from natural moulds, casts were whitened with ammonium chloride sublimate. a: Dorsal aspect of Sucocystis undata Friedrich, 1993, Holotype IGUR 1033a, middle Cambrian (Morocco), note the bulky marginal plates. Scale bar: 5 mm. b: Dorsal aspect of Sucocystis melendezi (Schroeder, 1973), Holotype GPM L51/4/20, middle Cambrian (Ateca, Zaragoza, Spain). Scale bar: 5 mm. c: Dorsal aspect of Sucocystis acrofera Friedrich, 1993, PIW 92V183, middle Cambrian (Montagne Noire, France). Scale bar: $5 \mathrm{~mm}$.d: Ventral aspect of Sucocystis theronensis Cabibel, Termier \& Termier, 1959, PIW 92V16II, middle Cambrian (Montagne Noire, France). Scale bar: $5 \mathrm{~mm}$. $\boldsymbol{e}, \boldsymbol{f}$ : Dorsal and lateral aspects of Sucocystis quadricornuta Friedrich, 1993, Holotype PIW 92 V51, middle Cambrian (Morocco), note the large dorsal swellings. Scale bars: $2 \mathrm{~mm}$. 
Además, considera que Elliptocinctus pertenece a los Gyrocystidae y que la presencia de dos ambulacros en Elliptocinctus, uno de ellos, el izquierdo, muy desarrollado es una característica primitiva de los Gyrocystidae.

Friedrich (1995) defiende la validez de la familia Sucocystidae por poseer características distintivas suficientes.

En este trabajo se acepta la propuesta de Friedrich (1993, 1995). La familia Sucocystidae tiene características diagnosticas reconocibles y que los diferencia de las otras dos familias Gyrocystidae y Trochocystitidae. Las principales son la longitud de los surcos epitecales, la ausencia de plataforma frontal (marginale Lippe, sensu Friedrich, 1993) y la posición de los túmulos en la cara ventral.

\section{Género Sucocystis Cabibel, Termier \& Ter- mier, 1959}

Especie tipo: Sucocystis theronensis Cabibel, Termier \& Termier, 1959

Géneros sinónimos: Trochocinctus Termier \& Termier (1973), Cyclocinctus Termier \& Termier (1973).

Observaciones: Friedrich (1993) ofrece una descripción morfológica detallada de todas las especies de este género, por lo que aquí únicamente se indicarán algunas cuestiones generales sobre su morfología.

La teca de Sucocystis presenta un contorno de circular a piriforme aunque es algo asimétrica (Figs. 2, 3). El lado derecho siempre está más desarrollado que el izquierdo. Se compone de un numero variable de placas marginales que puede ser 11 o 12, o más raramente 10 o incluso hasta 13 (Friedrich, 1993). El número de placas marginales suele variar mucho en algunas especies (i.e. Sucocystis theronensis) (Figs. 3, 4). Una de las principales características de este género es la ausencia de plataforma frontal (marginale Lippe, sensu Friedrich, 1993).

La boca se sitúa como en todos los cincta, entre las placas M1r y M2r. Todas las especies presentan un surco epitecal izquierdo que alcanza la placa M11, excepto la aquí descrita cuyo surco izquierdo llega hasta la M2l. El surco derecho puede faltar, estar extremadamente reducido o ser ligeramente más largo y alcanzar la placa M3r (Fig. 4). Todas las especies presentan un dintel compuesto por tres (más raramente cuatro) placas postoperculares. Algunas especies desarrollan grandes protuberancias dorsales en estas placas (i.e. Sucocystis theronensis en la placa central o $S$. quadricornuta en las laterales). El opérculo se dispone de manera casi vertical en todas las especies.

Una característica notable de las placas marginales es que algunas desarrollan engrosamientos laterales o protuberancias dorsales. Este es el caso de las placas M21, M3 y M4r en $S$. undata o, más notablemente, M31 y M4r en S. quadricornuta (Figs. 3a, e, f, 5).

Las placas supracentrales suelen ser muy pequeñas y muy ornamentadas. Sin embargo las infracentrales son muy grandes, extremadamente planas y sin ornamentación (Figs. 2, 3d).

Todas las especies presentan la cara inferior del cinctus ondulada como consecuencia del desarrollo de túmulos

Sucocystis theronensis

Sucocystis bretoni

Sucocystis melendezi

Sucocystis acrofera

Sucocystis undata

Sucocystis quadricornuta

S. sp. A

M61 M51 M41 M31 M21 M11 M0 M1r M2r M3r M4r M5r M6r
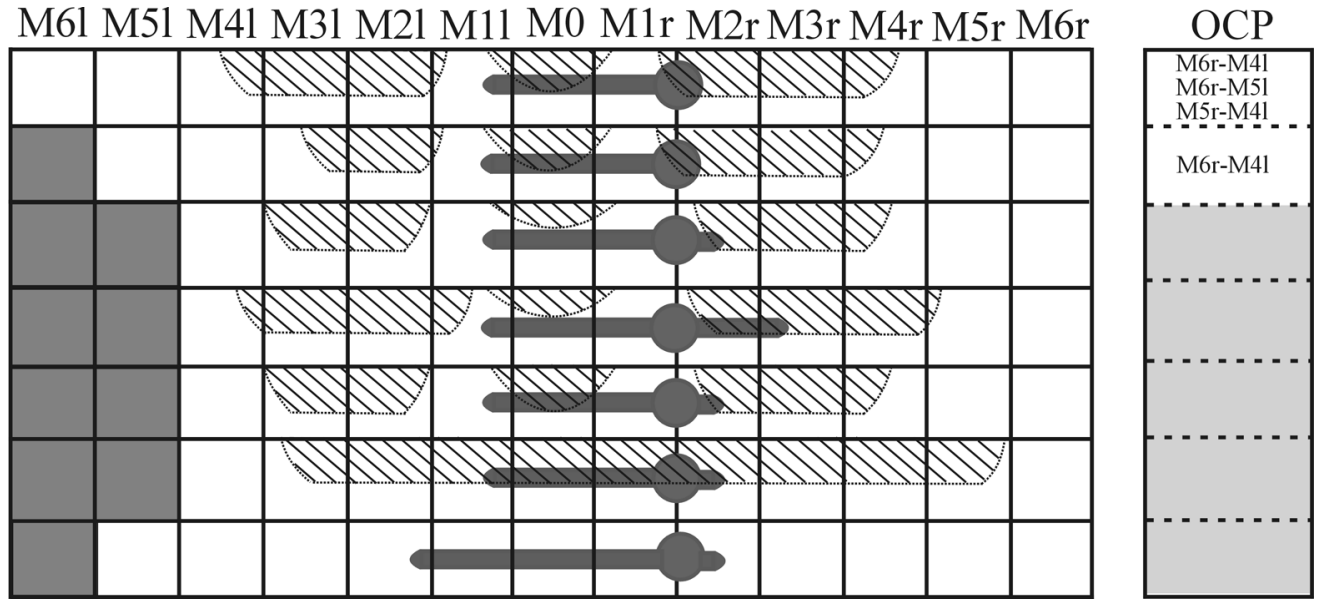

Figura 4. Disposición de los ambulacros, diagrama de placas marginales y posición de los túmulos ventrales en las especies del género Sucocystis. Superficie ventral en S. sp. A desconocida. En blanco = placas marginales presentes; círculo gris = posición de la boca; líneas gris oscuro = longitud de los surcos epitecales; rayas oblicuas = posición de los túmulos ventrales. OCP: Otras configuraciones posibles de placas marginales; M1-M6: placas marginales; 1: izquierda; r: derecha.

Schematic diagram showing the number of marginal plates comprising the cinctus, food groove distribution and ventral swelling development on marginal plates for different species of Sucocystis. Ventral surface in S. sp. A unknown. Open boxes = marginal plate present; grey circle = position of mouth; thick grey lines = length of left or right food grooves; and oblique hatching = presence of ventral swellings on marginal plates. Abbreviations: OCP, other possible plate arrangements; M1-M6, marginal plates; l, left; r, right. 
(tumulus, sensu Sdzuy, 1993) (Figs. 2, 3d). Generalmente, existe un túmulo desarrollado bajo la placa M0 que a veces se extiende lateralmente hacia las placas M1, otras veces puede faltar (i.e. S. quadricornuta) o estar muy reducido (i.e. S. acrofera). Además existen grandes plataformas ventrales (formadas por unión de varios túmulos) en las placas marginales.

La estela no está preservada en la mayoría de las especies aunque en ciertas especies es muy larga.

Especies reconocidas: Sucocystis theronensis y Sucocystis bretoni en la Formación Couloma en la Montaña Negra (Cámbrico medio, Francia), Sucocystis melendezi y $S$. acrofera de la misma región pero en la Formación La Gardie. S. melendezi se ha encontrado además en Ateca (Cadenas Ibéricas, España) en la Formación Borobia. S. quadricornuta procede Zona de Bailiella cf. levyi (Cámbrico medio) en el Lemdad-Synkline (Alto Atlas, Marruecos). Sucocystis undata procede de la Zona de Bailiella cf. levyi (Cámbrico medio), pero de la parte media del Alto Atlas, también en Marruecos. Existe un taxón asignado con dudas al género, $S$. ? maroccana de la Meseta Küs-
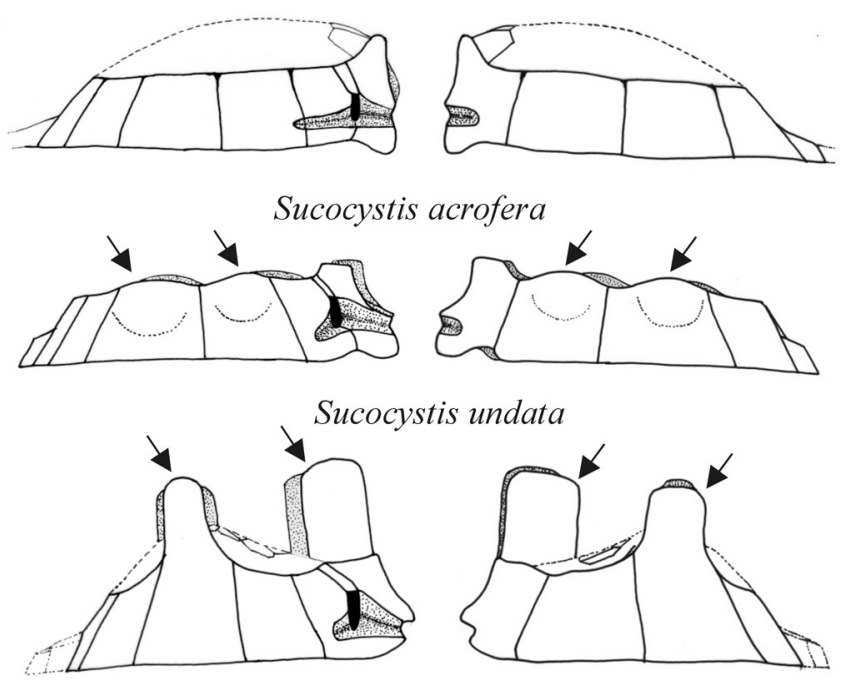

Sucocystis quadricornuta

Figura 5. Reconstrucciones en vista lateral de tres especies de Sucocystis (modificado de Friedrich, 1993). a: S. acrofera Friedrich, 1993, b: S. undata Friedrich, 1993, c: S. quadricornuta Friedrich, 1993. Observar la presencia/ausencia y desarrollo de protuberancias dorsales o engrosamientos laterales en las placas marginales (ver flechas) que son la principal diferencia entre las especies.

Reconstructions of three species of Sucocystis in lateral view (modified from Friedrich, 1993). a: S. acrofera Friedrich, 1993, b: S. undata Friedrich, 1993, $\boldsymbol{c}$ : S. quadricornuta Friedrich, 1993. Compare the development of dorsal protuberances on the marginals of different Sucocystis species (see arrows). ten en Marruecos. Además, existen una serie de taxones en nomenclatura abierta como son Sucocystis sp. S del Cámbrico medio de Cerdeña, $S$. aff theronensis del Languedociense inferior en la Montaña Negra (Francia) y $S$. aff. quadricornuta del Languedociense medio también en la Montaña Negra (Francia).

\section{Sucocystis sp. A Figs 6, 7}

Material y modo de conservación: Un solo ejemplar bien conservado que representa la cara dorsal del animal. La teca se encuentra completa y articulada. Las placas supracentrales se han colapsado sobre la infracentralia. La estela se encuentra parcialmente desarticulada. El ejemplar está conservado como molde externo.

Descripción: Teca de contorno piriforme, algo asimétrica, muy ancha en la parte anterior y más estrecha en la posterior. Las medidas máximas son $10 \times 8 \mathrm{~mm}$. La teca se compone de doce placas marginales (M6r-M51).

En la parte anterior derecha y entre las placas M1r y M2r se encuentra la boca que es bastante grande, de contorno ovalado y ligeramente deprimida. Las dimensiones son de $2 \mathrm{~mm}$ x $1 \mathrm{~mm}$ aproximadamente. De ella irradian dos surcos epitecales bastante profundos, uno izquierdo largo que llega a alcanzar el primer tercio de la placa M21, y otro derecho corto que ocupa casi todo el margen de la placa M2r. No existe plataforma frontal.

El complejo porta-opercular se compone del opérculo, dintel, porta y dos tubérculos adoperculares. El opérculo se encuentra colapsado y es bastante grande. Su contorno es piriforme y se articula al dintel sólo por su cara posterior. Los tubérculos adoperculares son muy reducidos y se sitúan sobre las placas M1; en su cara interna presentan las facetas de anclaje con el dintel (facetas adoperculares) que son también muy reducidas. El dintel se compone de cuatro placas postoperculares bastante grandes (hasta cuatro veces más grandes que las placas supracentrales), que son claramente reconocibles.

La placa marginal M0 tiene un tamaño muy reducido y sólo se observa su parte anterior que está totalmente ocupada por parte del surco epitecal izquierdo. Las placas M1 contienen un tubérculo adopercular cada una y están también ocupadas en su parte frontal por el surco epitecal izquierdo. La placa M2r presenta su parte superior modificada para la articulación con la placa supraoral, que no se ha conservado. Su cara frontal aparece ocupada por el surco epitecal derecho. El resto de las placas marginales son bastante parecidas entre sí, presentan plataforma marginal externa e interna desarrolladas y en su parte superior la articulación con la supracentralia. La parte posterior de las placas M6r y M51 se encuentra modificada para su articulación con la estela.

Sólo se conserva parte del tegumento supracentral que está desarticulado y colapsado sobre la infracentralia. En la parte anterior se observan claramente las cuatro placas postoperculares que constituirían el dintel. El resto de las supracentrales son parecidas entre sí, de muy pequeño tamaño (menos de 500 $\mu \mathrm{m})$ y ornamentadas.

La estela está incompleta y desarticulada. Sólo se observan placas esfenoides anchas y de gran tamaño; la primera esfenoide derecha aparece articulada con el cinctus. 
Discusión y Comparación: La asignación de este ejemplar al género Sucocystis viene justificada por el desarrollo de los surcos epitecales, uno derecho muy corto y otro izquierdo más largo y que alcanza la placa M21. Otras características son el desarrollo de dintel, la teca de contorno ligeramente asimétrico, ausencia de plataforma frontal y la presencia de placas supracentrales pequeñas, numerosas y ornamentadas.

El contorno general de la teca es diferente a otros Sucocystis, asemejándose únicamente a algunos ejemplares de S. theronensis (Friedrich, 1993: lám. 7 fig. 3). En el resto de las especies, los contornos suelen ser casi circulares con relaciones longitud/anchura que rondan el 1.

Por el número de surcos epitecales, Sucocystis sp. A se asemeja a $S$. melendezi, $S$. undata, $S$. quadricornuta y $S$. acrofera, pero se diferencia de estas especies en que el surco epitecal izquierdo de $S$. sp. A es ligeramente más largo y alcanza la placa M21.

El cinctus carece de engrosamientos laterales o protuberancias dorsales lo que diferencia a $S$. sp. A de especies como $S$. undata o $S$. quadricornuta. La estela en casi todas las especies es desconocida aunque en Sucocystis sp. A las esfenoides se asemejan bastante a las de S. bretoni.

Sucocystis sp. A presenta algunas características exclusivas, como la longitud del surco epitecal izquierdo, que es más largo que en otras especies, el contorno de la teca o el número de placas que componen el dintel. Ante la falta de más material se prefiere mantener el taxón en nomenclatura abierta de momento.

Localidad y edad: Mesones de Isuela (Zaragoza), Formación Murero, Cámbrico medio, Languedociense inferior, Zona de Solenopleuropsis thorali.

\section{IMPLICACIONES BIOSTRATIGRÁFICAS}

De todas las especies de Sucocystis conocidas únicamente se conoce con exactitud la posición estratigráfica
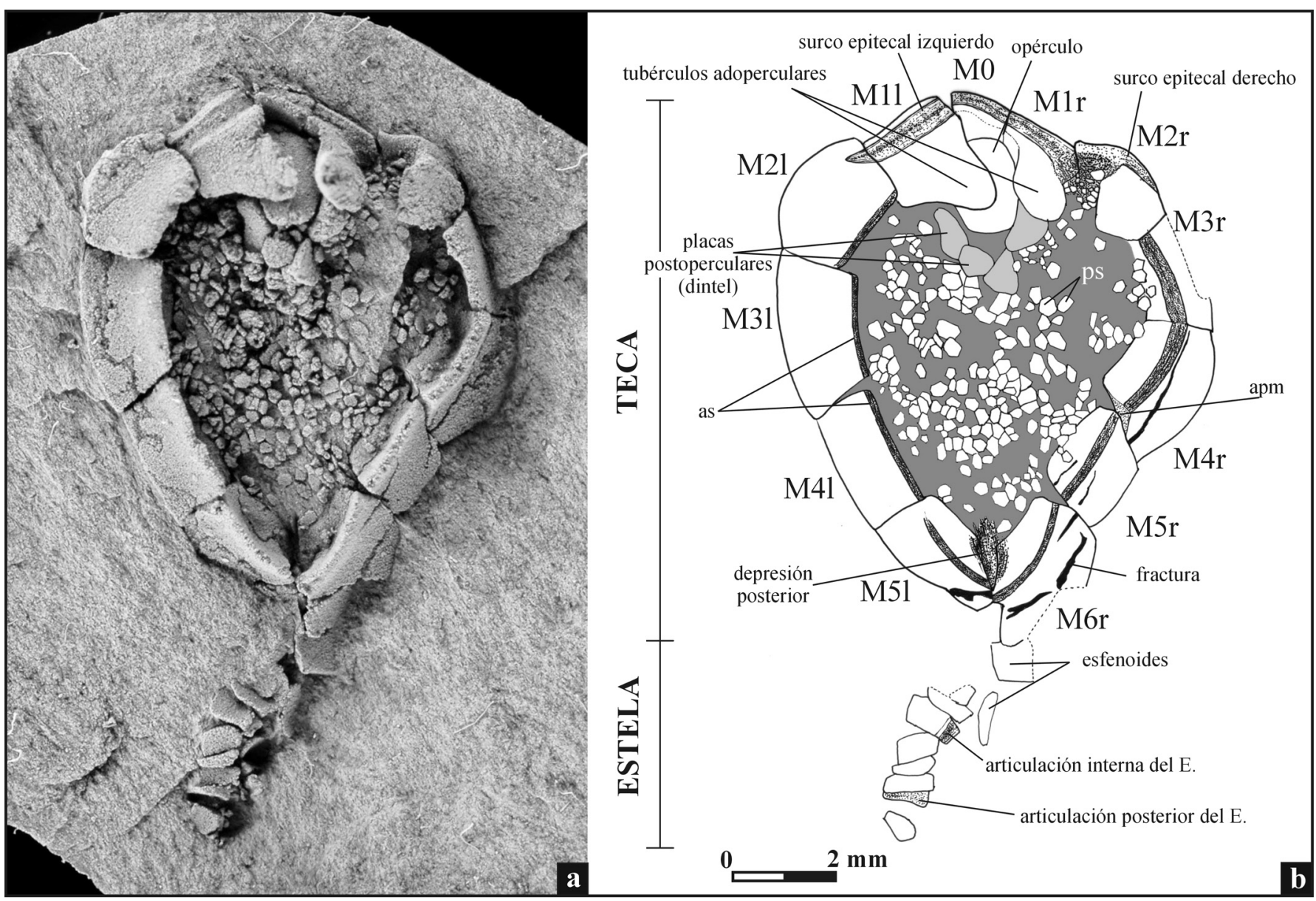

Figura 6. Fotografía (a) y dibujo a cámara clara (b) en vista dorsal de Sucocystis sp. A. MPZ2007/2479. Fotografía a partir de molde de látex teñido con cloruro amónico. Abreviaturas: M, placa marginal; 1, izquierda; r, derecha; as, articulación con la supracentralia; apm, articulación entre placas marginales; E, estela.

Photograph (a) and camera lucida drawing (b) of Sucocystis sp. A. MPZ2007/2479 in dorsal view. Photograph of latex cast taken from a natural mould, cast whitened with ammonium chloride sublimate. Abbreviations: $M$, marginal plate; $l$, left; $r$, right; as, supracentral articulation facet; apm, articulation between marginal plates; $E$, stele. 
del material procedente de la Montagne Noire (Francia) y de las Cadenas Ibéricas (España). La posición estratigráfica del material de Marruecos se conoce sólo a grandes rasgos, mientras que el nivel concreto del material procedente de Italia se desconoce.

En las Cadenas Ibéricas se han encontrado dos especies. Sucocystis melendezi, proviene de la parte alta del Grupo Acón, de niveles con trilobites (descritos por Shergold \& Sdzuy, 1991) equivalentes al Languedociense superior (Álvaro et al., 2001). Sucocystis sp. A. se ha encontrado en el techo de la Formación Murero, en la zona de Solenopleuropsis thorali y por tanto es de edad Languedociense inferior según la definición original del piso (Álvaro \& Vizcaïno, 1998).

Las especies de Francia proceden todas de la Montaña Negra en diferentes niveles. Según Vizcaïno \& Lefebvre (1999) y Álvaro et al. (2001), Sucocystis theronensis Cabibel, Termier \& Termier, 1959 procede de la Zona de Solenopleuropsis (Solenopleuropsis) y de la parte baja de la Zona de Jincella? cf. prantli de edades Languedociense inferior y medio respectivamente. Sucocystis bretoni Friedrich, 1993 proviene de la base de la Zona de Jincella? cf. prantli de edad Languedociense medio. Ambas especies han sido encontradas en la Formación Coulouma. S. acrofera Friedrich, 1993 proviene de la parte alta del Languedociense medio (Zona de Bailiella souchoni) y $S$. melendezi (Schroeder, 1973) de la parte alta del Languedociense medio y el superior (Zonas de B. souchoni y de Eccaparadoxides macrocercus). Ambas especies han sido encontradas en la Formación La Gardie.

Todos los ejemplares de Sucocystis quadricornuta Friedrich, 1993 proceden de la zona de Bailiella cf. levyi (sensu Geyer, 1990) en el Sinclinal de Lemdad (extremo oriental del Alto Atlas, Marruecos). Sucocystis undata Friedrich, 1993 procede de la misma zona biostratigráfica, pero en la región central del Alto Atlas (Marruecos). La zona de Bailiella cf. levyi, equivale en la Península Ibérica y Francia a los últimos niveles con Solenopleuropsis de edad Languedociense inferior (Álvaro et al., 2003).

El material procedente de Cerdeña de Sucocystis sp. S, fue recogido por el Profesor Hammann en la Formación Cabitza, en Iglesias-Gonnesa. Friedrich (1995) no indica el nivel concreto de procedencia, así que nos tenemos que guiar por la edad de esta formación, que en esta área incluye todo el Caesaraugustiense y el Languedociense (Loi et al., 1995; Álvaro et al., 1999).

Una vez vista la distribución de todas las especies de Sucocystis se concluye que una de las especie más antiguas es $S$. sp. A del Languedociense inferior de España. De edad similar son las especies de Marruecos, $\mathrm{Su}$ cocystis undata y $S$. quadricornuta. S. theronensis también aparece en el Languedociense inferior aunque, su distribución se extiende hasta el medio, en la Montaña Negra. Sucocystis bretoni y S. acrofera caracterizan el Languedociense medio en Francia. Sucocystis melendezi caracteriza bien la parte alta del Languedociense medio y el Languedociense superior en Francia por lo que su presencia en el Grupo Acón en las Cadenas Ibéricas, junto a trilobites del Languedociense superior, es coherente con su distribución en Francia (Shergold \& Sdzuy, 1991; Álvaro et al., 2001). Finalmente, el taxón en nomenclatura abierta de Cerdeña permanece sin ubicación estratigráfica precisa.

\section{IMPLICACIONES FILOGENÉTICAS}

Uno de los principales problemas en el estudio de los cincta es el establecer criterios diagnósticos exclusivos para las diferentes familias. Friedrich (1993) fue el primero en evidenciar este problema indicando que, comparados con otros equinodermos, los cincta son una clase con unas autapomorfías claras, pero a su vez falta de caracteres diagnósticos claros con los que diferenciar dis-
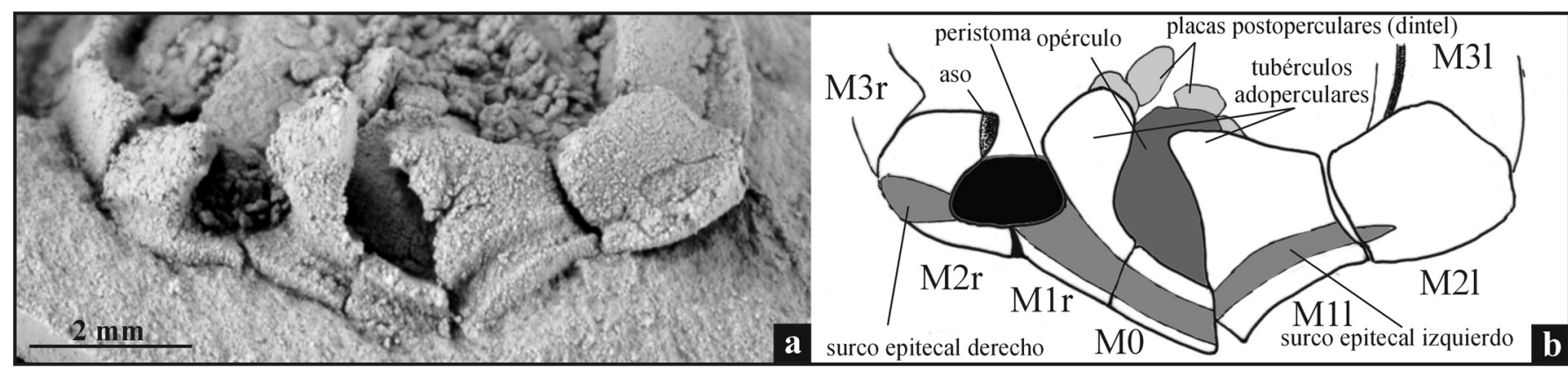

Figura 7. Fotografía (a) y dibujo a cámara clara (b) en vista frontal de Sucocystis sp. A. MPZ2007/2479. Fotografía a partir de molde de látex teñido con cloruro amónico. Abreviaturas: M, placa marginal; 1, izquierda; r, derecha; aso, articulación con la placa supraoral.

Photograph (a) and camera lucida drawing (b) of Sucocystis sp. A. MPZ2007/2479 in frontal view. Photograph of latex cast taken from a natural mould, cast whitened with ammonium chloride sublimate. Abbreviations: $M$, marginal plate; $l$, left; $r$, right; aso, supraoral articualation facet. 


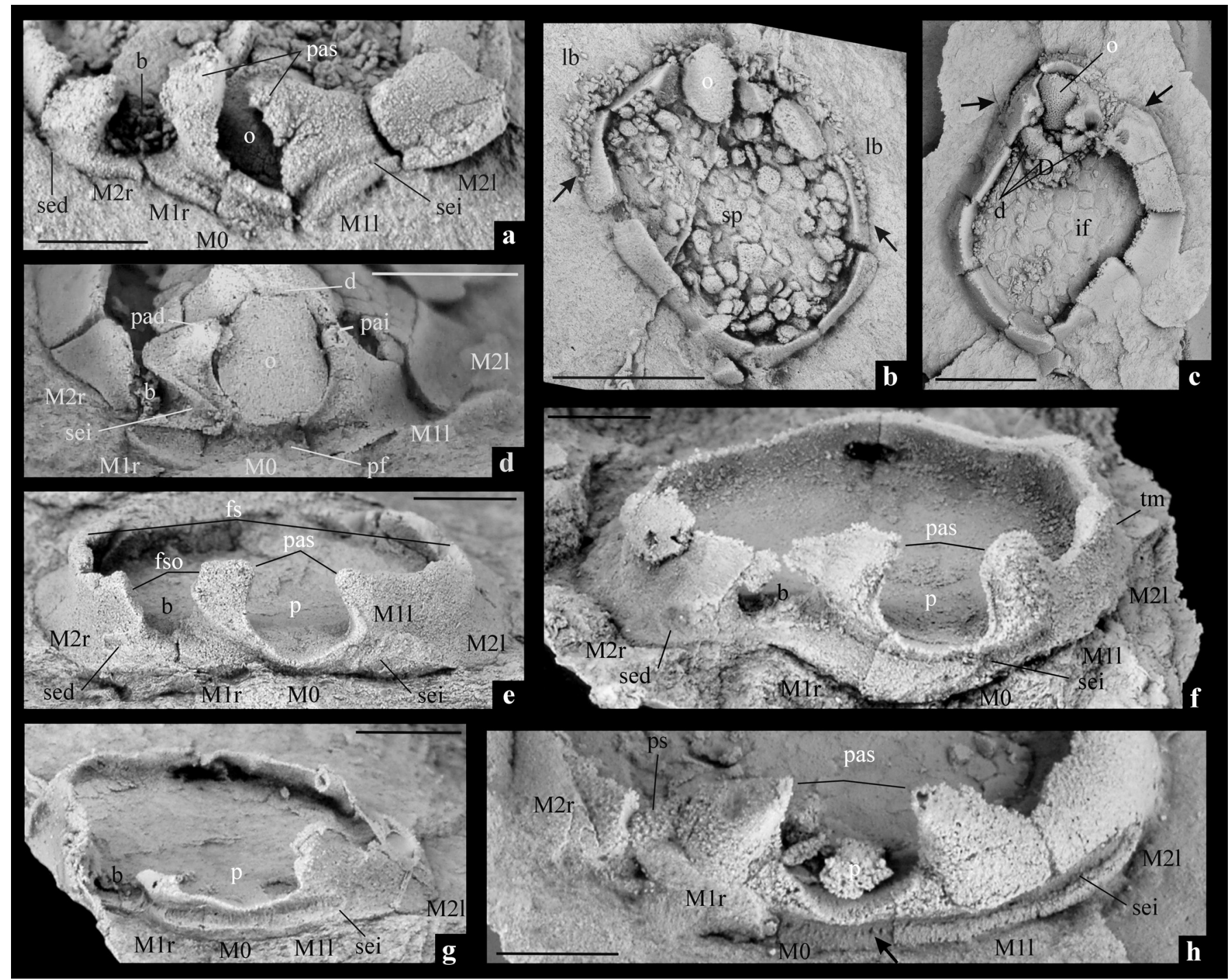

Figura 8. Diferentes configuraciones de los surcos epitecales en distintos cincta. Todos los ejemplares han sido fotografiados a partir de moldes en látex teñidos con cloruro amónico. a: Sucocystis sp. A, MPZ2007/2479. Barra: 2 mm. b: Sotocinctus ubaghsi Sdzuy 1993, MPZ2008/3. Barra: 5 mm. c: Elliptocinctus barrandei (Munier-Chalmas \& Bergeron 1889), NHM E 7696. Barra: 5 mm. d: Gyrocystis testudiformis Friedrich, 1993, MPZ2008/4. Barra: 3 mm. e: Sucocystis melendezi (Schroeder 1973), Holotipo GPM L51/4/20. Barra: 3 mm. f: Sucocystis undata Friedrich 1993, Holotipo IGUR 1033a. Barra: 3 mm. g: Sucocystis acrofera Friedrich 1993, Holotipo PIW 92V43a. Barra: 3 mm. h: Trochocystites bohemicus Barrande 1887 PIW 92V1. Barra: $3 \mathrm{~mm}$. Abreviaturas: b, boca; d, dintel; fs, faceta con la supracentralia; if, infracentralia; 1, izquierda; lab, labro; M, placa marginal; o, opérculo; pas, procesos adoperculares; pai, proceso adopercular izquierdo; pad, proceso adopercular derecho; pf, plataforma frontal; p, porta; ps, placa supraoral; r, derecha; sei, surco epitecal izquierdo; sed, surco epitecal derecho; sp, supracentralia.

The lengths of marginal grooves in different species of cinctans. All photographs are of latex casts taken from natural moulds, casts were whitened with ammonium chloride sublimate. a: Sucocystis sp. A, MPZ2007/2479. Scale bar: 2 mm. b: Sotocinctus ubaghsi Sdzuy 1993, MPZ2008/3. Arrows indicate the ends of marginal grooves, Scale bar: $5 \mathrm{~mm}$. $\mathrm{c}$ : Elliptocinctus barrandei (Munier-Chalmas \& Bergeron, 1889), NHM E 7696. Scale bar: 5 mm. Arrows indicate the ends of marginal grooves. d: Gyrocystis testudiformis Friedrich, 1993, MPZ2008/4. Scale bar: 3 mm. e: Sucocystis melendezi (Schroeder, 1973), Holotype GPM L51/4/20. Scale bar: 3 mm.f: Sucocystis undata Friedrich, 1993, Holotype IGUR 1033a. Scale bar: $3 \mathrm{~mm}$. g: Sucocystis acrofera Friedrich, 1993, Holotype PIW 92V43a. Scale bar: $3 \mathrm{~mm}$. h: Trochocystites bohemicus Barrande, 1887, PIW 92V1. Scale bar: $3 \mathrm{~mm}$. Abbreviations: M, marginal plate; l, left; r, right; o, operculum; pas, adopercular processes; pai, left adopercular process; pad, right adopercular process; $b$, mouth; sei, left food groove; sed, right food groove; lab, labrum; sp, supracentralia; $d$, lintel; if, infracentralia; $f$, supracentral articulation facet; pf, broad anterior shelf; $p$, porta; ps, supraoral plate. 
tintos grupos o familias. De hecho, una de las principales discusiones existentes en la taxonomía de los cincta trata sobre la existencia de dos o tres familias (ver discusión en Friedrich, 1993, 1995; Sdzuy, 1993).

La principal característica diferenciadora entre ellas, es el número y longitud de los surcos epitecales (Ubaghs, 1968; Friedrich, 1993). Los cincta pueden presentar uno o dos surcos epitecales. Estos surcos surgen de la boca y, en vida, estuvieron cubiertos por multitud de pequeñas placas que conformaron una estructura llamada labro. La familia Trochocystitidae se caracteriza por la presencia de dos surcos epitecales largos que sobrepasan la mitad del contorno de la teca, la Sucocystidae por un surco epitecal izquierdo medio y uno derecho ausente o muy reducido y la Gyrocystidae por un único surco izquierdo muy reducido. Ya que todos los cincta más antiguos conocidos [i.e. Sotocinctus ubaghsi Sdzuy, 1993 (ver Fig. 8b), Asturicystis jaekeli Sdzuy, 1993 o Trochocystoides planus Sdzuy, 1993] presentan dos surcos epitecales largos, Sdzuy (1993), planteó la hipótesis de que la condición ancestral fuera esta. El criterio estratigráfico no es a priori el mejor para conocer si una estructura es primitiva o derivada. De hecho el cincta más antiguo conocido hasta la fecha presenta un único surco epitecal izquierdo Rahman y Zamora (en prensa).

La ontogenia de los cincta tampoco ofrece por el momento ninguna solución para conocer cual fue la condición primitiva de los surcos epitecales en los cincta. $\mathrm{Al}$ menos en las colecciones existentes de cincta, no se ha observado ninguna variación en la longitud y número de los surcos entre individuos de distinto tamaño.

Para tratar de esclarecer la condición primitiva de los surcos epitecales en los cincta hay que estudiar la misma característica en otros grupos de equinodermos. De todos los equinodermos primitivos conocidos, los ctenocystoideos son el grupo que más se parece superficialmente a los cincta (Friedrich, 1993; Jefferies et al., 1996; DomínguezAlonso, 1999; David et al., 2000). En ambos, un anillo de placas marginales se encuentra cubierto por dos membranas de placas. La boca en ambos se abre lateralmente a través de dicho anillo. Mientras que los cincta presentan surcos alimenticios que se dirigen a la boca, en los ctenocystoideos, a partir de la boca, irradia hacia izquierda y derecha el ctenidio (series de pequeñas placas que cubren la parte anterior, protegiendo la gran cavidad anterior). Estas estructuras han sido interpretadas como surcos alimenticios protegidos por placas coberteras y que se dirigen hacia la boca (Robison \& Sprinkle, 1969; Domínguez-Alonso, 1999). Las placas del labro de los cincta y las placas ctenidiales de los ctenocystoideos son estructuras homólogas (Domínguez-Alonso, 1999). La disposición del ctenidio es simétrica mientras que el labro que cubre los surcos epitecales es asimétrico. La condición ancestral en los cincta fue seguramente la de dos surcos epitecales ya que esta es la condición existente en los ctenocystoideos, y por tanto es seguramente la que también tuvo el ancestro común de ambos.

A lo largo de la evolución de los cincta se observa una reducción de los surcos epitecales en longitud y número. Esta reducción se produce seguramente de manera independiente en las diferente familias de cincta. En la familia Gyrocystidae, por ejemplo, este proceso conlleva la reducción total del surco epitecal derecho y el acortamiento del izquierdo (i.e. Gyrocystis testudiformis, Fig. 8d).

De las siete especies reconocidas en el género Sucocystis cinco presentan dos surcos epitecales. Esta condición se observa también en otros géneros como Trochocystites o Sotocinctus (Fig. 8b), aunque en estos, los surcos son mucho más largos. De todas ellas $S$. acrofera (Fig. 8g) presenta un surco derecho más largo que otras especies alcanzando la placa marginal M3r y $S$. sp. A (Fig. 8a) uno izquierdo más desarrollado que alcanza la placa M21. Especies como $S$. quadricornuta, $S$. undata (Fig. 8f) o $S$. melendezi (Fig. 8e) experimentan una reducción de ambos surcos, de manera que el izquierdo alcanza sólo la placa M11 y el derecho es muy reducido, alcanzando la primera parte de la placa M2r. Por último $S$. theronensis y $S$. bretoni ya no presentan surco epitecal derecho. Esta gran variación en longitud y número de los surcos epitecales parece indicar que Sucocystis (sensu Friedrich, 1993) es probablemente un grupo parafilético.

\section{AGRADECIMIENTOS}

En primer lugar queremos expresar nuestro agradecimiento a Jorge Colás (Universidad de Zaragoza) por encontrar el ejemplar estudiado en este trabajo. A los Drs. Gerd Geyer (Instituto Paleontológico de Würzburg, Alemania), Bertrand Lefebvre (Universidad Claude Bernard, Lyon, Francia), Markus Bertling (Universidad de Münster, Alemania), Andrew B. Smith y Richard Jefferies (ambos del Natural History Museum, Londres), se les agradece enormemente el acceso al material previamente estudiado por otros investigadores y depositado en sus respectivas instituciones. El excelente trabajo de los revisores, Dra. Monica Martí Mus (Universidad de Extremadura) y Dr. Patricio Domínguez (Universidad Complutense, Madrid) ha mejorado el manuscrito. También agradecemos la labor del editor, Dr. Rodolfo Gozalo.

El programa Europa de la CAI ha subvencionado las visitas del primer firmante a los centros de Alemania y Londres para revisar colecciones. Este trabajo es una contribución al Proyecto Consolíder CGL2006-12975/BTE («MURERO») del Ministerio de Educación y Ciencia de España, y al Grupo Consolidado E-17 («Patrimonio y Museo Paleontológico») de la Consejería de Ciencia, Tecnología y Universidad del Gobierno de Aragón. El primer autor (S. Z.) goza de una beca predoctoral financiada por el Departamento de Ciencia, Tecnología y Universidad del Gobierno de Aragón. IR agradece al National Environmental Research Council (NER/S/A/2005/13593) por el soporte financiero. Por último, agradecer a Isabel Pérez Urresti, por sus fotografías del material. 


\section{BIBLIOGRAFÍA}

Aliaga, A. 1968. Geologische untersuchungen in den Ostlichen Iberischen ketten nördlichdes río Jalón (Zaragoza, Spanien). Inaugural-Dissertation, Universität Münster, 99 pp. (inédito).

Álvaro J. J. \& Vizcaïno D. 1998. Révison biostratigraphique du Cambrien moyen du versant méridional de la Montagne Noire (Languedoc, France). Bulletin de la Société Géologique de France, 169, 233-242.

Álvaro J. J., Vizcaïno, D. \& Vennin E. 1999. Trilobite diversity patterns in the Middle Cambrian of southwestern Europe: a comparative study. Palaeogeography, Palaeoclimatology, Palaeoecology, 151, 241-254.

Álvaro J. J., Lefebvre, B., Shergold, J. H. \& Vizcaïno, D. 2001. The Middle-Upper Cambrian of the southern Montagne Noire. Annales de la Société Géoloique du Nord, $2^{\text {ème }}$ série, 8, 205-211.

Álvaro, J.J., Elicki, O., Geyer, G., Rushton, A.W.A., \& Shergold, J.H. 2003. Palaeogeographical controls on the Cambrian trilobite immigration and evolutionary patterns reported in the western Gondwana margin. Palaeogeography, Palaeoclimatology, Palaeoecology, 195, 5-35.

Barrande, J. 1887. Système Silurien du centre de la Bohème. Vol. VII. Classe des échinodermes, ordre des Cystidées. Praga-Leipzig, 233 pp.

Bather, F. A. 1930. A Class of Echinoderma without trace of radite symmetry. Archivio Zoologico Italiano, 14, 431-439.

Bottjer, D. J., Davidson, E. H., Peterson, K. J., \& Cameron, R. A. 2006. Paleogenomics of echinoderms. Science, 314, 956-960.

Cabibel, J., Termier, H. \& Termier, G. 1959. Les échinodermes mesocambriens de la Montagne Noire (sud de la France). Annales de Paléontologie, 44, 281-294.

Carls, P. 1983. La Zona Asturoccidental-Leonesa en Aragón y el Macizo del Ebro como prolongación del Macizo Cantábrico. In: Contribuciones sobre temas generales (coord. J. A. Comba). Instituto Geológico y Minero de España, Libro Jubilar de J. M. Ríos, 3, 11-32.

Clausen, S. 2004. New Early Cambrian eocrinoids from the Iberian Chains (NE Spain) and their role in nonreefal benthic communities. Eclogae Geologicae Helvetiae, 97, 371-379.

David, B., Lefebvre, B., Mooi, R., \& Parsley, R. 2000. Are homalozoans echinoderms? An answer from the extraxialaxial theory. Paleobiology, 26, 529-555.

Dies, M.E., Gozalo, R. \& Liñán, E. 2004. Zonación del límite Bilbiliense-Leoniense (Formación Valdemiedes, Cámbrico Inferior-Medio) en las Cadenas Ibéricas. Geo-Temas, 6 (2), 283-286.

Donayre, F. M. 1873. Bosquejo de una descripción física y geológica de la Provincia de Zaragoza. Memorias de la Comisión del Mapa Geológico de España, 1, 126 pp.

Domínguez-Alonso, P. 1999 The early evolution of echinoderms: the class Ctenocystoidea revisited. In: Echinoderm Research 1998 (eds C. Carnevalli \& F. Bonasoro). Rotterdam, Balkema. 263-268.
Domínguez, P., \& Jefferies, R. P. S. 2005. A cladogram for the Deuterostomia based on molecular-biological and fossil evidence. In: Abstracts of the $53^{\text {rd }}$ suymposium on vertebrate palaeontology and comparative anatomy (ed. P. M. Barrett). The Natural History Museum, London, 30, 30.

Friedrich, W. P. 1993. Systematik und Funktionsmorphologie mittelkambrischer Cincta (Carpoidea, Echinodermata). Beringeria, 7, 3-190.

Friedrich, W. P. 1995. Neue Nachweise mittelkambrischer Cincta (Carpoidea, Echinodermata) aus Marokko, Sardinien und Süd-Wales. Beringeria, sp. Issue 2, 255-269.

Geyer, G., 1990. Revised Lower to lower Middle Cambrian biostratigraphy of Morocco. Newsletter on Stratigraphy, 22, 53-70.

Gil Cid, M. D. \& Domínguez, P. 1995. Presencia de Gyrocystis Jaekel,1918 en el Cámbrico Medio de Zafra (Badajoz). Revista de la Sociedad Geológica de España, 8, 99-110.

Gozalo, R. \& Liñán, E. 1988. Los materiales hercínicos de la Cordillera Ibérica en el contexto del macizo ibérico. Estudios geológicos, 44, 399-404.

Gozalo, R., Álvaro, J., Liñán, E., Sdzuy, K. \& Truyols, J. 1993. La distribucion de Paradoxides (Acadoparadoxides) mureroensis Sdzuy, 1958 (Cambrico Medio basal), y sus implicaciones paleobiogeográficas. Cuaderno do Laboratorio Xeolóxico de Laxe, 18, 217-230.

Jaekel, O. 1900. Ueber Carpoideen: eine neue Klasse von Pelmatozoen. Zeitschrift der Deutschen Geologischen Gesellschaft, 52, 661-667.

Jaekel, O. 1918. Phylogenie und System der Pelmatozoen. Paläontologische Zeitschrift, 3, 1-128.

Jefferies, R. P. S. 1990. The solute Dendrocystoides scoticus from the Upper Ordovician of Scotland and the ancestry of chordates and echinoderms. Palaeontology, 33, 631-679.

Jefferies, R. P. S., Brown, N. A., \& Daley, P. E. J. 1996. The early phylogeny of chordates and echinoderms and the origin of chordate left-right asymmetry and bilateral symmetry. Acta Zoologica, 77, 101-122.

Josopait, V. 1972. Das Kambrium und das Tremadoc von Ateca (Westliche Iberische Ketten, NE-Spanien). Münsterche Forschungen zur Geologie und Paläontologie, 23, 1-121.

Liñán, E. 1983. Una nueva hipótesis sobre le estructura geológica del basamento al NE de la Península Ibérica. $V$ Asamblea Nacional de Geodesia y Geofísica. Resúmenes, 283.

Liñán E., Villas, E., Gámez Vintaned, J. A., Álvaro, J., Gozalo, R., Palacios, T. \& Sdzuy, K. 1996. Síntesis Paleontológica del Cámbrico y Ordovícico del Sistema Ibérico (Cadenas Ibéricas y Cadenas Hespéricas). Revista Española de Paleontología, $\mathbf{N}^{\mathbf{0}}$ extraordinario, 21-32.

Loi, A., Pillola, G.L. \& Leone, F., 1995. The Cambrian and Early Ordovician of southwestern Sardinia. Rendiconti del Seminario della Facoltà di Scienze dell'Università di Cagliari, Suppl., 65, 63-81.

Lotze, F. 1929. Stratigraphie und Tektonik des Keltiberischen Grundgebirges (Spanien). Abhandlungen der Gesellschaft der Wissenschaften zu Göttingen, mathematisch-physikalische Klasse, neue Folge, 14 (2), 1-320. 
Lotze, F. 1961. Das Kambrium Spaniens. Teil I: Stratigraphie. Akademie der Wissenschaften und der Literatur, Abhandlungen der mathematisch-naturwissenschaftlichen Klasse, 1961 (6), 1-216.

Olmo, P., Hernández, A., Aragonés, E., Aguilar M. J. \& Ramírez del Pozo, J. 1981. Mapa y Memoria de la hoja $n^{\circ} 381$ (Illueca) del mapa geológico de España, escala 1:50000. Instituto Geológico Minero de España, Madrid, 1-39.

Parsley, R. L. 1999. The Cincta (Homostelea) as blastozoans. In: Echinoderm Research (eds M. D. Candia Carnevali \& F. Bonasoro). A. A. Balkema, Rotterdam, 369-375.

Palacios, P. 1893. Reseña geológica de la región meridional de la provincia de Zaragoza. Boletín de la Comisión del Mapa Geológico de España, 19, 1-112.

Rahman, I. A. \& Zamora, S. en prensa. The oldest cinctan carpoid (stem-group Echinodermata) and the evolution of the water vascular system. Zoological Journal of the Linnean Society.

Robison, R. A. \& Sprinkle, J. 1969. Ctenocystoidea. New class of primitive echinoderms. Science, 166 (3912), 1512-1514.

Schmitz U. 1971. Stratigraphie und Sedimentologie im Kambrium und Tremadoc der Westlichen Iberischen Ketten nördlich Ateca (Zaragoza), NE Spanien. Münstersche Forschungen zur Geologie und Paläontologie, 22, 123 pp.

Schmidt-Thomé, M. 1973. Beiträge zur Feinstratigraphie des Unterkambriums in der Iberischen Ketten (NordostSpanien). Geologische Jahrbuch, Reihe B, 7, 3-43.

Schröeder, R. 1973. Carpoideen aus dem Mittelkambrium Nordspaniens. Palaeontographica Abt. A., 141, 119-142.

Sdzuy, K. 1961. Das Kambrium Spaniens. Teil II: Trilobiten. Akademie der Wissenschaften und der Literatur, Abhandlungen der mathematisch-naturwissenschaftlichen Klasse, 1961 (7-8), 499-690 (217-408).

Sdzuy, K. 1993. Early Cincta (Carpoidea) from the Middle Cambrian of Spain. Beringeria, 8, 189-207.

Shergold, J.H. \& Sdzuy, K. 1991: Late Cambrian trilobites from the Iberian Mountains, Zaragoza Province, Spain. Beringeria, 4, 193-235.
Smith, A. B. 2005. The pre-radial history of echinoderms. Geological Journal, 40, 255-280.

Termier, H. \& Termier, G. 1973. Les Échinodermes Cincta du Cambrien de la Montagne Noire (France). Geobios, 6, 243-266.

Ubaghs, G. 1968. Homostelea. In: Treatise on Invertebrate Paleontology. Echinodemata (ed. R. C. Moore). Geological Society of America and University of Kansas Press, Boulder, 565-581.

Ubaghs, G. 1971. Diversité et spécialisation des plus anciens échinodermes que l'on connaisse. Biological Reviews, 46, 157-200.

Ubaghs, G. 1975. Early Palaeozoic echinoderms. Reviews in Earth and Planetary Science, 3, 79-98.

Valenzuela J. I., Gámez, J. A., Liñán, E. \& Sdzuy, K. 1990. Estratigrafía de la región de Brea. Cadena Ibérica Oriental. Boletín de la Real Sociedad Española de Historia Natural (Sección Geología), 85, 45-54.

Vizcaïno, D. \& Lefebvre, B. 1999: Les échinodermes du Paléozoïque inférieur de Montagne Noire: biostratigraphie et paléodiversité. Geobios, 32, 353-364.

Zamora, S., Liñán, E., Gámez Vintaned J. A., Domínguez Alonso, P. \& Gozalo, R. 2007a. Nuevo carpoideo de la clase Cincta Jaekel, 1918 del norte de España: inferencias sobre la morfología funcional del opérculo. Ameghiniana, 44, 727-738.

Zamora, S., Liñán, E., Domínguez Alonso, P., Gozalo, R. \& Gámez Vintaned, J. A. 2007b. A Middle Cambrian edrioasteroid from the Murero biota (NE Spain) with Australian affinities. Annales de Paléontologie, 93, 249-260.

Zamora, S. \& Smith, A. B. 2008. A new Middle Cambrian stem-group echinoderm from Spain: Palaeobiological implications of a highly asymmetric cinctan. Acta Paleontologica Polonica, 53, 207-220.

Manuscrito recibido: 10 de Abril, 2008 Manuscrito aceptado: 30 de Septiembre, 2008 